\title{
Deux visions du bonheur selon la nature chez Camille Lemonnier et Georges Eekhoud
}

Dans les dernières années du XIX ${ }^{\mathrm{e}}$ siècle, l'écrivain belge Camille Lemonnier compose trois romans rattachés au mouvement naturiste, L'̂̂le vierge, Adam et Ėve et Au cour frais de la forêt, qui mettent en scène l'utopie d'un retour à la nature ${ }^{1}$. Son compatriote Georges Eekhoud publie en 1912, après 14 années de travail, Les Libertins d'Anvers, long roman historique consacré aux hérésies du Moyen Âge et de la Renaissance dans sa ville natale d'Anvers. Eekhoud y fait l'apologie d'une libération des mœurs à travers le récit de l'aliénation d'un peuple par un christianisme intransigeant, appelant indirectement de ses vœux l'avènement d'une société future moins puritaine et plus tolérante. Comme le remarque Clément Dessy, le roman d'Eekhoud porte un regard poétique sur l'histoire. Malgré un évident travail de documentation, Eekhoud ne propose pas un récit objectif, mais plutôt une reconstitution fantasmée du passé. Son approche est avant tout émotionnelle et a pour but de "faire ressortir l'esprit d'Anvers ", tout un héritage spirituel capable d'influencer les consciences (Dessy, 2016, p. 231). Les romans de Lemonnier et d'Eekhoud ont en commun le rêve d'un âge d'or, qui correspond soit à l'aube de l'humanité sur une île ou dans une forêt édéniques, soit à une période légendaire de l'histoire, leur but étant de faire entrevoir un mode de vie libéré du joug de la civilisation. Les deux écrivains se nourrissent des aspirations de toute une génération marquée par une fin de siècle où le spectre de la décadence fait peu à peu place à la volonté d'une régénération profonde de la société, qui se rattache à un fort sentiment nationaliste. Ils sont soumis à des influences communes,

Philippe Chavasse - professeur associé à l'Institut de technologie de Rochester. Adresse de correspondance : Department of Modern Languages and Cultures, College of Liberal Arts, Rochester Institute of Technology, 92 Lomb Memorial Drive, Rochester, NY 14623-5604, États-Unis ; e-mail : pxcgsl@rit.edu

ORCID iD : https://orcid.org/0000-0002-6431-2543

1. Sur le naturisme, mouvement littéraire né en France à la fin du XIX ${ }^{e}$ siècle, voir l'étude de MartinSchmets (1984), qui évoque la genèse de ce mouvement et retrace son évolution en Belgique. 
les mythes fondateurs du récit national de leur pays, ou encore le socialisme qui imprègne les lettres belges à la fin du XIX ${ }^{\mathrm{e}}$ siècle. Néanmoins, ils expriment des visions différentes de ce que devrait être la société idéale. Lemonnier, conformément à la pensée traditionaliste des poètes naturistes, imagine un monde dans lequel l'homme et la femme occupent une fonction utilitaire dictée par la nature, qui les incite à travailler pour le bien de la communauté et à procréer afin de perpétuer l'espèce. Se servant du modèle offert par les hérésiarques anversois et se situant dans le courant de la pensée libertaire, Eekhoud prêche au contraire une foncière oisiveté et une jouissance inconditionnelle du corps, qui fait éclater le carcan imposé par le couple et la famille. Lemonnier et Eekhoud se rejoignent toutefois dans leur approche de la femme, qui reste dans leurs œuvres au service de l'homme, tout en étant adulée comme un guide indispensable ou comme une sainte.

\section{L'identité belge à la fin du XIXe siècle : mise au point sur les fondements de l'utopie}

Lemonnier et Eekhoud sont incontestablement les deux grands écrivains réalistes belges de la fin du XIX ${ }^{\mathrm{e}}$ siècle. Ils appartiennent à la génération qui participe au réveil artistique de la Belgique dans les années 1880 visant à constituer une littérature nationale émancipée du modèle français ${ }^{2}$. Sous l'impulsion du naturalisme zolien, Lemonnier donne l'exemple en invitant ses compatriotes à cultiver les traits qui les rattachent au sol où ils sont nés. L'observation minutieuse d'un moment et d'un milieu, et la recherche de marques héréditaires associées à un déterminisme de race revêtent pour les Belges de l'époque un caractère identitaire. Au fameux "Soyons-nous " de La Jeune Belgique, Lemonnier répond par " Nous-mêmes ou périr » et Eekhoud par " être soi-même ", les deux romanciers prêchant tour à tour une authenticité qui est un appel à rester fidèle aux sources vives de l'inspiration des artistes belges (Gorceix, 1997, p. 18, 17, 24). La descente au fond d'eux-mêmes à laquelle se livrent les écrivains belges de la fin de siècle s'accompagne d'une remontée dans le temps, le récit identitaire se trouvant consolidé par le prestige de l'histoire nationale. Dans la Belgique du XIX ${ }^{e}$ siècle, l'identité nationale est définie en grande partie en référence à un âge d'or, le $\mathrm{XVI}^{e}$ siècle, période qui est encore marquée par l'influence du Moyen Âge, et dont lévocation s'accompagne d'une stéréotypie que l'on retrouve dans les romans de Lemonnier et d'Eekhoud ${ }^{3}$. Celle-ci est associée à la représentation du Belge

2. Sur le réveil artistique de la Belgique, voir l’ouvrage de synthèse de Dumont (1991, p. 69-77). Voir aussi la présentation de la Belgique fin de siècle par Gorceix (1997, p. 13-53).

3. Sur le XVI e siècle comme mythe fondateur de la Belgique, voir les deux articles de Quaghebeur $(2002,2005)$. Voir aussi l'article de Soncini sur la médiévalisation de la littérature belge de langue française au XIX ${ }^{\mathrm{e}}$ siècle. Soncini explique qu'une distorsion se produit dans cette littérature qui "superpose le Moyen Âge au matériau flamand pris en dehors ou au-delà du Moyen Âge même » (2004, p. 2). Elle 
comme un irréductible homme du Nord, à la fois travaillé par des appétits charnels démesurés et capable de faire preuve d'une bonté et d'une innocence évangéliques ${ }^{4}$. Figuration du bon sauvage, le Belge apparait comme porteur d'une promesse de rédemption passant par le retour à un état primordial contre les ravages causés par une civilisation qui dénature lêtre humain. Pour des écrivains belges comme Lemonnier et Eekhoud, le rêve d'une terre promise, terre de bonheur, se rattache à des mythes fondateurs de la belgitude comme la figure du gueux associée au $\mathrm{XVI}^{e}$ siècle et à la révolte menée par Guillaume d'Orange contre Philippe II d'Espagne ${ }^{5}$. Symbole du patriotisme belge, le gueux est un homme fruste, proche de la terre, un réfractaire profondément épris de justice et de liberté, intraitable contre les forces qui cherchent à l'opprimer, doux, farceur, épicurien, un cœur simple, enthousiaste, capable d'extase mystique au contact avec une nature qui lui révèle la présence de Dieu. Les personnages des romans de Lemonnier et d'Eekhoud se rapprochent des gueux par leur panthéisme et leur aspiration à une liberté sans frein qui s'accompagne d'un rejet de la propriété privée et d'un égalitarisme conforme à la nature.

Ce souci d'égalité fait écho aux importants conflits sociaux qui se déroulent en Belgique dans les vingt dernières années du XIX ${ }^{e}$ siècle et ont un impact considérable sur la littérature 6 . La veine réaliste ou naturaliste est largement façonnée par ces conflits. Elle participe à l'expression de l'anti-bourgeoisisme viscéral qui caractérise les écrivains de la génération de Lemonnier et Eekhoud. Leurs préoccupations sociales sont conditionnées par le choc qu'occasionne dans le pays une industrialisation rapide s'accompagnant de la paupérisation des campagnes, qui est à l'origine d'un exode rural massif et de la prolifération dans les banlieues des métropoles d'un prolétariat et d'un sous-prolétariat soumis à des conditions de vie difficiles.

Les romans de Lemonnier et d'Eekhoud que nous proposons d'étudier dans cet article tentent d'apporter une réponse à l'exploitation des masses laborieuses par une élite égoïste et puritaine en prêchant une liberté primordiale. Pour Lemonnier, cela passe par une adhésion au mouvement naturiste, dans lequel l'écrivain belge retrouve l'importance du déterminisme héréditaire propre au naturalisme zolien, mais assorti d'un élan régénérateur, un optimisme viscéral mis au service de la cause nationale ${ }^{7}$. Eekhoud, quant à lui, reprend le mythe familier du XVI ${ }^{e}$ siècle et se focalise sur une hétérodoxie religieuse qui a inspiré des philosophes anarchistes tels que le Belge

donne comme exemple La Légende d'Ulenspiegel de Charles De Coster, qui baigne entièrement dans une atmosphère médiévale bien que son histoire se déroule en pleine Renaissance.

4. Sur le "Mythe nordique ", voir l'ouvrage de Gonne consacré au travail de feuilletoniste d'Eekhoud. Gonne énumère les principaux éléments de ce mythe qui constitue un topos de la littérature belge jusqu'à la première guerre mondiale (2017, p. 27).

5. Quaghebeur rappelle que le gueux était un « mythe national » omniprésent dans la littérature belge au XIX ${ }^{e}$ siècle (2005, p. 36).

6. Pour un survol de la situation économique de la Belgique au XIX siècle, consulter Luc (1990, p. 9-14). Voir aussi l'étude d'Aron (1985) sur le socialisme dans les lettres belges.

7. Sur les liens entre le naturisme et le naturalisme, voir l'article de Christie (1963). 
Raoul Vaneigem, auteur de la préface de l'édition des Libertins d'Anvers parue en 2009, et son homologue français Michel Onfray, tous deux adeptes de la pensée de Charles Fourier. Tout comme Eekhoud, Fourier, Vaneigem et Onfray ont rêvé une société meilleure, plus égalitaire, qui se rapproche de la vie et de la nature et prenne en compte les instincts de l'être humain au lieu de les bafouer. Vaneigem, qui avoue avoir découvert l'histoire des hérésiarques anversois en lisant le roman d'Eekhoud, leur a consacré deux ouvrages, Le Mouvement du Libre-Esprit et La Résistance au christianisme, ouvrages repris par Onfray dans son Christianisme hédoniste. Il sera intéressant de montrer dans quelle mesure la filiation du naturisme pour Lemonnier et le lien avec un anarchisme qui érige la jouissance et le plaisir en valeurs suprêmes dans le cas d'Eekhoud amènent les deux écrivains belges à envisager la « réhabilitation des pulsions de vie » de manières distinctes (Vaneigem, 2009, p. 12).

\section{Base idéologique de la société à laquelle aspirent Lemonnier et Eekhoud}

L'aspiration à l'avènement d'une ère nouvelle, où la liberté de l'homme et de la femme pourrait enfin s'affirmer conformément à la nature, incite Lemonnier et Eekhoud à poser dans leurs œuvres un regard critique sur la société qui les entoure, et à essayer de la déstabiliser par l'intermédiaire de romans qui remettent en cause les fondements de cette société. Cela leur vaudra, au cours de l'année 1900, d'être poursuivis l'un après l'autre par la justice suite à la publication de L'Homme en amour et d'EscalVigor, deux ouvrages accusés de pornographie ${ }^{8}$. Dans L'Homme en amour, Lemonnier fustige une société puritaine qui détourne l'homme de l'instinct et de la nature. Quant à Eekhoud, il fait l'apologie dans Escal-Vigor des amours masculines au détriment de l'hétérosexualité présentée comme un asservissement des corps et des âmes, leur soumission à une logique mercantile qui a pour but la reproduction de l'espèce. Les deux écrivains belges seront acquittés, mais ils garderont de cette expérience une profonde amertume. Les amours masculines resteront le principal moteur des romans d'Eekhoud, mais l'écrivain se gardera désormais d'en faire ouvertement l'éloge. À l'évocation de relations homosexuelles, il substituera celle d'une homosocialité masculine mêlée à un hymne au terroir et à la patrie, allant jusqu'à reprendre dans L'Autre vue la théorie de la terre et des morts soutenue par Maurice Barrès afin de justifier son désir de communion panthéiste avec les hommes qu'il aime'. Lemonnier, pour sa part, approfondira son rêve d'une humanité réconciliée avec la nature, mais sur un mode moins polémique que dans L'Homme en amour, en proposant une ma-

8. Sur le procès d'Escal-Vigor, voir l'article de Detemmerman (1984), qui fait aussi référence au procès de L'Homme en amour. Plus récemment, le procès d'Escal-Vigor a été commenté par Dupont (2015) et Rosenfeld (2018).

9. Sur les accents barrésiens de L'Autre vue, voir mon article dans French Forum (Chavasse, 2022). 
nière de vivre réalisée à l'écart des villes et de la civilisation, dans un espace situé hors du temps et dépourvu de localisation géographique précise.

Les deux romans que Lemonnier écrit à la suite de L'Homme en amour sont Adam et Ève et Au cour frais de la forêt. Ceux-ci nous projettent dans un monde de la régénération, le monde de la forêt, au sein d'une nature vierge chargée de symboles, dans laquelle l'homme et la femme sont à nouveau à même d'entendre la voix de l'instinct. Les Libertins d'Anvers n'est pas le premier roman d'Eekhoud publié après Escal-Vigor. Il y a eu entre-temps L'Autre vue, mais le projet monumental qu'est la composition des Libertins d'Anvers, vaste fresque historique, est commencé en 1898, un an avant la parution d'Escal-Vigor et deux ans avant le procès ${ }^{10}$. Eekhoud y poursuit la recherche d'une légitimation de ses penchants homosexuels en plongeant dans le Moyen Âge et, surtout, la Renaissance, période de l'histoire qu'il affectionne tout particulièrement à cause de la liberté des mœurs qui la caractérise ${ }^{11}$. Il évoque différentes figures d'hérésiarques associées au passé de sa ville natale, Anvers, et se compose ainsi une généalogie selon sa nature, justifiant indirectement ses tendances homosexuelles considérées comme déviantes au moyen d'une hérédité qui ancre son vice dans un sol et une race qu'il glorifie. Cela lui permet de produire un discours déculpabilisant. Même si la Réforme protestante et la Contre-réforme menée par les catholiques écrasent finalement les hérésies qui remettent en question l'orthodoxie chrétienne, le fondement identitaire est clairement établi. La pudibonderie qui incite à jeter l'anathème sur des comportements sexuels irréguliers est présentée comme une contrainte arbitraire dans Les Libertins d'Anvers, l'imposition d'une manière d'être contre-nature et, par conséquent, vouée à disparaître. Au centre du roman d'Eekhoud rayonne une figure de martyr, Éloi Pruystinck, fondateur de la secte des Loïstes, " aimable prophète d'un nouvel âge d'or, $[\ldots]$ apôtre de la lumière et du sourire ", qui prêche un panthéisme assez proche de celui adopté par les personnages de Lemonnier dans ses romans naturistes (1912, p. 377).

Paru en 1897, Lîle vierge de Lemonnier aborde déjà le thème du retour à la nature que l'on retrouve quelques années plus tard dans Adam et Ève et Au cour frais de la forêt. Toutefois, l'utopie présentée dans ce roman est nettement moins aboutie que dans les deux romans naturistes qui succèdent à L'Homme en amour. L'antagonisme entre d'un côté une existence édénique conforme à la nature et de l'autre la corruption des corps et des âmes par une civilisation urbaine mortifère est fortement marqué dans L'Île vierge, qui se situe tantôt sur une île au décor de pastorale, où « une paix merveilleuse baigne la savane, la fraternité des hommes et des troupeaux

10. La première publication de L'Autre vue date de 1904. Ce roman sera réédité par la suite sous le titre Voyous de velours ou L'Autre vue.

11. L'intérêt d'Eekhoud pour la Renaissance se manifeste non seulement dans Les Libertins d'Anvers, mais aussi dans l'attention marquée que l'écrivain accorde à l'Angleterre du XVI siècle. Voir à ce sujet l'article de Dessy (2020). Sur l'homosexualité d'Eekhoud, voir les travaux de Lucien (1999). 
comme au temps d'Arcadie » et où l'on vit sans la conscience du péché originel, tantôt dans une cité lugubre, où " [i]l pleut une cendre d'heures lentes et tristes ", saturée par la présence de la mort et peuplée d'hommes et de femmes soumis à la morale du christianisme (1896, p. 34, 155). Deux approches de la vie sont clairement opposées dans L'T̂le vierge, une approche païenne et une approche chrétienne, qui serviront de base à l'utopie mise en scène dans Adam et Ève et Au cour frais de la forêt, l'idéal recherché se situant entre les deux, dans un état de nature magnifié par une bonté évangélique qui fait écho au sacrifice de Jésus Christ sur la croix.

Les figures christiques abondent dans l'œuvre d'Eekhoud, que ce soient Henry de Kehlmark et Guidon Govaertz dans Escal-Vigor, Laurent Paridael dans L'Autre vue, ou encore Éloi dans Les Libertins d'Anvers. Le héros eekhoudien se retrouve volontiers sacrifié, sa mort s'accompagnant généralement d'une révélation censée déciller les yeux de la masse aveugle et assurer sa conversion à une religion vouée au culte de la nature, de la beauté et de l'authenticité. Cette religion doit remplacer l'ancienne, à savoir le christianisme dogmatique qui domine la culture occidentale. C'est dans Les Libertins d'Anvers qu'Eekhoud développe avec le plus d'ampleur le modèle qu'il souhaiterait voir triompher. Il décrit minutieusement les tribulations de sectes hérétiques qui sont autant d'avatars de la société dont il rêve, une société foncièrement libre dans laquelle chaque individu obéit à un instinct de vie tout en se laissant guider par son cœur. Ce monde idéal, dont Eekhoud entrevoit des manifestations dans les époques antérieures, est nourri par les deux grandes traditions spirituelles qui se sont succédées dans l'histoire de l'Occident, le paganisme et le christianisme, et emprunte à chacune ce qu'il y a de meilleur.

La convergence du paganisme et du christianisme présente dans les romans naturistes de Lemonnier et dans Les Libertins d'Anvers d'Eekhoud se rattache à l'engouement des écrivains belges du XIX ${ }^{e}$ siècle pour le Moyen Âge. Le Moyen Âge est certes en vogue à cette époque aussi bien en France qu'en Allemagne; toutefois, comme le souligne Anna Soncini, « quand on aborde la littérature belge, le discours se modifie car on touche à l'archétype même de cette littérature, qui trouve en cela son unité profonde » (2004, p. 1). Il existe une coïncidence entre l'imaginaire flamand et le Moyen Âge, celui-ci correspondant pour les écrivains belges francophones, dont la plupart étaient de souche flamande, à «la représentation d'un monde intérieur qui dit la force de l'imaginaire flamand depuis toujours" (Soncini, 2004, p. 2) $)^{12}$. Ce "monde intérieur ", qui puise aux racines de l'âme belge, est incarné par le mystique du XIV siècle Jan Ruysbroeck, que Maurice Maeterlinck fait découvrir à ses compatriotes grâce à sa traduction en français de L'Ornement des noces spirituelles. Les écrits de Ruysbroeck fascinent les écrivains belges, qui y voient une source d'inspiration familière. Ils s'en imprègnent, mettant en scène dans leurs œuvres, comme le font Lemonnier et Eekhoud, le problème fondamental posé au Moyen Âge du « choix entre une vie

12. Sur le rôle déterminant de l'héritage flamand pour les écrivains belges de la fin du XIX siècle, voir les remarques de Gorceix (1998, p. 25-27). 
païenne et une vie catholique, une vie sexuelle et une vie chaste» (Soncini, 2004, p. 7). Les mondes qu'évoquent Lemonnier et Eekhoud sont ainsi marqués par une dualité entre un instinct irrépressible de vie et des passions mortifères associées à une société dite civilisée qui mène lêtre humain à sa perte. Les deux écrivains finissent par prêcher un panthéisme qui ancre l'homme et la femme dans la matière, leur permettant de recouvrer leur force originelle, tout en faisant d'eux des voyants sensibles au miracle de la création et capables de marcher dans la voie tracée par leurs ancêtres.

\section{Hérédité et élan vitaliste dans L'île vierge, Adam et Ève, Au cœur frais de la forêt et Les Libertins d'Anvers}

Lîle vierge met en scène une communauté idéale fondée à l'écart de la ville. Cette microsociété vit repliée sur elle-même dans une sorte de jardin d'Éden appelé Éolie. Son noyau se compose de Sévère Barba et ses quatre enfants, auxquels viennent s'ajouter les paysans qui travaillent sur l'île et les mendiants de passage invités à la table des Barba. Le roman de Lemonnier oppose deux modes de vie apparemment irréconciliables, d'un côté l'existence sereine des habitants d'Éolie qui vivent en harmonie avec la nature, et de l'autre le calvaire des habitants de la ville qui sont dominés par le dogme chrétien, la conscience du péché et l'obsession de la mort rédemptrice. L'œuvre de Sévère sur Éolie doit réparer la faute ancestrale en faisant table rase du passé et en se tournant résolument vers la vie. Sévère invite son entourage à célébrer la beauté du monde et à cultiver un bonheur qui passe par le respect des autres et de la nature, et par un travail quotidien accompli de plein gré en se mettant au service de la communauté. L'île où vivent Sévère et ses enfants est associée à l'antiquité et au paganisme. La ville baigne au contraire dans une atmosphère moyenâgeuse, fortement empreinte de christianisme.

La confrontation entre ces deux espaces introduit un conflit entre des forces opposées, qui finit par ruiner l'œuvre de Sévère en détruisant l'innocence nécessaire à la pérennité de la société idéale créée sur Éolie. La menace qui pèse sur la jeune génération des Barba est liée à leur hérédité, ce "péché de démence et de luxure qui dévasta la race des Barba » (1896, p. 10). Malgré tous les efforts de Sévère pour l'éradiquer, elle ressurgit inéluctablement. Peu à peu au cours du roman est révélé le secret de l'inceste commis par le frère et la sœur de Sévère, qui a abouti à la naissance d'Élée, que celui-ci a élevée comme sa propre fille. Cette révélation déclenche chez Élée et son cousin Sylvan un trouble associé à un instinct qui se manifeste par de la violence. Les règles établies par Sévère afin de protéger ses enfants, la pratique du végétarianisme et le commandement de ne pas tuer les animaux, volent alors en éclat. Sylvan, "mâle impétueux et primordial ", tue plusieurs bêtes qui peuplent le bois d'Éolie (1896, p. 128). Il ne parvient pas à contrôler la pulsion sanguinaire qui l'habite. Il essaie de se rapprocher du Dieu des chrétiens, vénéré par son cousin Éleuthère. Il cherche à sélever, à échapper à son aveuglement de païen prisonnier 
d'une immanence qui le confine à la bestialité. Il aspire à la révélation de la bonté évangélique du Christ, mais l'alliance entre le paganisme et le christianisme reste dans L'Île vierge une proposition inachevée. L'animalité de l'homme et de la femme est présentée comme une tare, la source d'un déséquilibre irrémédiable.

Par contraste avec L'̂lle vierge, Adam et Ėve et Au coeur frais de la forêt offrent des récits qui proposent une vision résolument optimiste de l'avenir, fondée sur un dépassement du péché de la race humaine. Dans les deux romans, Lemonnier raconte l'histoire d'un homme et d'une femme qui trouvent refuge dans une forêt où ils réapprennent à vivre et fondent une famille. Les deux récits comportent des circonstances légèrement différentes, mais les épreuves que traversent les couples sont sensiblement les mêmes. Dans un premier temps, ils sont grisés par l'instinct qui se réveille en eux au contact avec la nature, un instinct qui les amène à tuer les animaux de la forêt pour se nourrir. La bête sanguinaire qui se déchaîne en eux est mue par des pulsions qu'ils doivent apprendre à contrôler. Le travail et le fait de devenir parents leur permettent de s'élever au-dessus des désirs qui menacent de les submerger. L'homme devient bâtisseur, cultivateur et chef de famille, tandis que la femme s'occupe du foyer et des enfants. Loin des perversions de la ville, le couple mène une existence paisible en harmonie avec la nature. Il redécouvre les gestes des ancêtres et apprend à marcher dans la voie de la Providence.

Tout comme dans les romans de Lemonnier, la vie et la mort s'affrontent dans Les Libertins d'Anvers, et la mort finit par l'emporter. Le roman d'Eekhoud raconte le destin tragique d'hérésiarques confrontés à l'orthodoxie chrétienne. Le vent de liberté qui souffle sur la ville d'Anvers au Moyen Âge et à la Renaissance est interrompu par la Réforme protestante et la Contre-réforme qui répriment avec férocité les chrétiens dissidents. L'enjeu de la répression n'est pas simplement de mater une rébellion. Il s'agit de faire perdre son âme à une communauté tout entière. Eekhoud insiste sur la légitimité des hérésies qui ont eu lieu à Anvers et associe celles-ci au tempérament des Flamands. En retraçant l'histoire d'hérésies chrétiennes, il raconte l'histoire de sa ville natale depuis sa fondation jusqu'au XVIII ${ }^{e}$ siècle. Les deux sont inextricablement liées. Comme le souligne Eekhoud au début du roman : «De temps immémorial [...] Anvers fut un foyer de libertinage, voire d'anarchisme érotique [et] ne cessa de fomenter les hétérodoxies » (1912, p. 7). L'écrivain décrit dans le dernier chapitre de son livre les poursuites judiciaires qui aboutissent à l'exécution des Loïstes. À l'acharnement de Marie de Hongrie s'ajoute la machine implacable d'un État servile, la truculence et le libre-esprit faisant place à un puritanisme imposé à la population par la classe dominante. Les Libertins d'Anvers se conclut sur la fin d'un rêve qui coïncide avec l'écrasement de ce qui constitue l'âme d'un peuple.

Eekhoud est, comme Lemonnier, à la recherche d'un juste milieu entre un paganisme englué dans la matière et un christianisme éthéré qui permette de revitaliser la société en lui insufflant un élan salutaire. Il le trouve dans un panthéisme qui réconcilie le corps et l'esprit et est en harmonie avec l'âme du peuple flamand. L'évocation des hérésies chrétiennes n’a pour but ni de faire la promotion du vice, ni de démora- 
liser le lecteur qui, à l'aube du $\mathrm{XX}^{\mathrm{e}}$ siècle, peut être encore sensible au spectre de la décadence et à l'idée d'une dégénérescence de la race. Eekhoud oppose à cela le rappel insistant d'une histoire qui met en évidence un héritage qu'il convient aux habitants d'Anvers de se réapproprier. Car le récit détaillé des hérésies est finalement beaucoup plus prégnant que celui de leur répression. En rappelant à ses compatriotes des épisodes saillants de leur histoire, Eekhoud effectue un devoir de mémoire. Il commémore des morts héroïques, comme celle d'Éloi, et réactive un patrimoine commun. La leçon des Libertins d'Anvers est semblable à celle que l'on trouve dans les romans de Lemonnier, à savoir l'exhortation à un retour aux sources vives de l'existence et la recherche d'un équilibre qui permette de maîtriser la part d'ombre de la nature humaine, équilibre qui réside dans le respect de l'autre et, plus généralement, la pratique de la bonté évangélique.

Cette part d'ombre, associée à la cruauté dont l'homme et la femme sont capables, les personnages des romans de Lemonnier et d'Eekhoud y font face de manières très différentes. Alors que Lemonnier préconise une forme d'ascèse qui repose sur la vertu du travail et de la famille, conformément aux préceptes énoncés par le naturisme, Eekhoud propose, à travers l'exemple des hérésiarques de sa ville natale, un modèle fondé sur une doctrine du plaisir, qui prescrit le droit de chaque individu à jouir sans entraves de son corps selon une logique proche de la pensée anarchiste. La transformation de la société passe pour Lemonnier par une canalisation des désirs mis au service d'une communauté ayant pour base la famille, formée par un couple hétérosexuel et ses enfants. Il s'agit au contraire pour Eekhoud de faire éclater cette structure traditionnelle. La part d'ombre de l'être humain dans Les Libertins d'Anvers est ainsi extériorisée. La faute n'est pas le fait du personnage principal, Éloi, mais réside dans le regard des autres, ceux qui adhèrent à l'orthodoxie religieuse promue par les réformistes et les Jésuites, ou encore à l'orthodoxie sociale soutenue par la bourgeoisie puritaine.

\section{Les leçons du naturisme}

Le naturisme des romans de Lemonnier parus dans les dernières années du XIX ${ }^{\mathrm{e}}$ siècle a été dûment relevé par la critique ${ }^{13}$. Le poète, romancier et dramaturge français, Saint-Georges de Bouhélier, fondateur du mouvement naturiste, salue Lemonnier comme un écrivain naturiste. Il souligne le caractère prophétique de ses derniers romans, L'̂̂le vierge, L'Homme en amour, Adam et Ève et Au cour frais de la forêt, dans lesquels Lemonnier se détourne du réalisme et revient aux " époques premières " (1900, p. 252). Dans sa préface à un recueil de morceaux choisis de Bouhélier,

13. Voir notamment les ouvrages de Bazalgette (1904), Des Ombiaux (1909) et Rency (1922) sur l'œuvre de Lemonnier, et l'article de Krzywkowski (1997) consacré à ses romans naturistes. 
Lemonnier exprime son admiration pour ce dernier, qu'il considère comme un poète visionnaire. À travers les qualités qu'il attribue à l'œuvre de Bouhélier se dessine tout un programme, dans lequel on retrouve les idées chères aux écrivains belges de l'époque, en particulier le souci identitaire et l'intérêt pour la question sociale. Lemonnier insiste sur le fait que Bouhélier ne coupe pas l'homme de ses racines, du mystère insondable de sa genèse et de son destin, et prêche dans ses poèmes un égalitarisme, un sentiment de profonde fraternité, de communion entre les êtres : " il a pris parti pour les humiliés et les douloureux ; son art subtil, fleuri et raffiné qu'il dédia aux dieux heureux, il le voue à l'humanité opprimée. [...] Et il penche le front, il l'incline vers la conjecture du triste problème social» (1907, p. 17-18). Le monde dont rêve Bouhélier n'est pas un monde de compétition où les hommes se divisent l'ombre et la lumière et cherchent à se dominer entre eux. C'est un monde où la qualité humaine se mesure à l'aune du travail, un travail conforme à la nature et aux injonctions de la race. Car selon l'écrivain naturiste : "Un humain est le fils du fleuve, de la plaine et des monts : il s'accorde avec la terre, la fleur, la faune, la race ; il porte en soi toute la durée illimitée des ancêtres » $\left(1907\right.$, p. 11) ${ }^{14}$. Bouhélier formule le rêve d'une humanité qui renoue avec ses racines, composant des œuvres voisines de l'épopée, où le simple laboureur fait figure de héros moderne.

Les microsociétés imaginées par Lemonnier reposent sur un égalitarisme visible notamment dans les rapports entre les hommes et les femmes. Dans Adam et Ėve, l'homme traite la femme comme une égale. Il la laisse décider de son sort et de ses inclinations. Il attend qu'elle le rejoigne de plein gré dans la forêt, qu'elle fasse le premier pas pour lui signifier son désir de s'accoupler avec lui, qu'elle décide si elle préfère rester avec lui plutôt que de suivre le bel étranger qui vient leur rendre visite. De même, dans Au cœur frais de la forêt le personnage principal, Petit Vieux, laisse sa compagne, Frilotte, libre de choisir le cours de son existence. Il respecte sa volonté. Même s'il est dévoré de jalousie, il la laisse se faire courtiser par un équarrisseur, Iacq, « jeune géant » dont le physique agréable séduit les jeunes filles (1900, p. 173). Il la suit lorsqu'elle émet le souhait de retourner vivre dans la forêt, et il attend patiemment qu'elle fasse le premier pas avant de l'embrasser. Le respect de la femme s'étend à la création tout entière dans les romans de Lemonnier. Le commandement de ne pas tuer les animaux dans Lîle vierge correspond au mode de conduite que finissent par adopter les personnages d'Adam et Ève et Au cour frais de la forêt lorsqu'ils réalisent le caractère sacré de la vie. Ils s'éveillent alors à une conscience cos-

14. Le culte que Bouhélier voue aux ancêtres et à la nature fait bien sûr penser au culte barrésien de la terre et des morts. Comme Barrès, les écrivains naturistes appartiennent à la génération qui, au tournant du siècle, rêve de transformer le monde, l'imagination littéraire, comme le souligne Pierre Citti, « pass[ant] du dilettantisme égotiste et cérébral à l'exigence d'action et de responsabilité » (1987, p. 113). Un formidable élan vitaliste s'oppose alors à la décadence fin de siècle. Sur ce sujet, se reporter au livre de Citti, en particulier le chapitre 7 sur la notion d'énergie. 
mique se traduisant par la révélation du lien intime qui unit les différentes parties de la création.

La fraternité et la charité découlent de la communion avec la nature. La table des Barba est toujours ouverte aux vagabonds de passage. Le couple d'Adam et Ève ouvre sa maison aux démunis, partageant équitablement ses maigres ressources. Petit Vieux et Frilotte font de même avec le vieillard qu'ils rencontrent dans la forêt, cet homme dont " [l]a barbe avait mangé [le] visage jusqu’aux sourcils », et qui " avait de clairs yeux d'enfant» (1900, p. 215). Après un premier mouvement de peur, ils ouvrent leur cœur au vieillard et vivent quelques temps auprès de lui en parfaite harmonie. Ils se joignent par la suite à un peuple de forbans avec qui ils partagent le fruit de leur travail, un travail motivé non pas par le désir de s'enrichir, mais par la volonté de se mettre au service de la communauté. Le monde auquel rêve Lemonnier est un monde d'où la spéculation est absente. L'argent n'y a pas cours, et les richesses se limitent à ce qui est strictement nécessaire.

Si Lemonnier et Bouhélier se font les partisans des déshérités, les écrivains naturistes gardent néanmoins leurs distances vis-à-vis du socialisme. Bouhélier s'érige contre une forme d'art social qui donne l'illusion que le bonheur réside dans l'accession aux biens matériels. Il croit au contraire que « [d]e la quantité de beauté, dégagée par chacun de nous, dépendent notre allégresse et l'harmonie du monde " (1899, p. 79). Bouhélier déclare : "Le sentiment de leur forte beauté intrinsèque rendra joyeux les hommes, qui, jusquà ce jour, se sont habitués à mettre toute leur félicité dans la fortune, les matérialités. De l'idéal ! de l'idéal ! ouvrez profondément l'esprit du peuple, que le souffle de Dieu l'éclaircisse, accroissez le sens du sublime ! ( (1899, p. 81). Le rôle du poète naturiste est de galvaniser les énergies afin de régénérer l'âme de tout un peuple. Cette régénération passe par une prise de conscience de la beauté du monde et de la grandeur de chaque être humain qui se manifeste par des gestes simples accomplis chaque jour, comme ceux du paysan qui cultive son champ, dur labeur mis au service de la famille et de la communauté. Le but du naturisme est d'offrir des "spectacles civiques » et de glorifier la vie quotidienne, rudimentaire, vouée à des tâches essentielles, afin d'insuffler un sentiment d'héroïsme et de sacré (Bouhélier, 1899, p. 80).

Les personnages des romans naturistes de Lemonnier découvrent la valeur du travail, un travail nécessaire, conforme à leur instinct et leur destinée. La vie simple dont ils font l'expérience dans la forêt ou sur l'île d'Éolie les ramène dans le droit chemin qui a été oublié par les hommes civilisés. Ils prennent conscience qu'ils sont en train d'accomplir une tâche éternelle, celle du laboureur qui pousse péniblement sa charrue afin de préparer la récolte qui donnera à manger à sa femme et ses enfants, ou bien celle du charpentier qui bâtit une maison pour abriter les siens. La chaîne des générations est présente de manière insistante dans les trois romans naturistes de Lemonnier, où les personnages ne se réalisent pleinement que lorsqu'ils finissent par se plier au rôle qui leur est assigné depuis la nuit des temps, prendre une femme, ancrer son existence dans un labeur nécessaire à la survie de l'espèce, et procréer 
afin de répondre à l'injonction de la nature ${ }^{15}$. La leçon fondamentale des romans de Lemonnier est que toute vie participe à un éternel recommencement, un flot continu dans lequel s'enchaînent la mort et la génération. L'homme qui accomplit son devoir marche dans les traces de ses ancêtres. Il devient sensible à leur présence invisible qui vibre dans son corps et dans la création tout entière. La présence des ancêtres se fait sentir par exemple dans Adam et Ėve lorsque les deux protagonistes conçoivent leur premier enfant, cet acte les situant hors du temps en les intégrant au cycle de la vie :

Une grande paix nous enveloppait, un silence venu du fond des races autour de nous, comme si les ancêtres se tenaient droits derrière les arbres, un doigt sur la bouche. Nous avions mal divinement, nous ne savions pas si nous vivions encore ou si déjà c’était la délicieuse mort, comme une vie délivrée et infinie. (1899, p. 68)

Dans Les Libertins d'Anvers, la race est omniprésente. Son évocation à travers l'histoire de la ville d'Anvers correspond à l'expression d'un mythe des origines. Tout comme Lemonnier, Eekhoud est à la recherche d'une authenticité qui garantisse une forme de liberté totale permettant d'échapper aux mauvais conditionnements responsables de l'égarement de la race. Bouhélier considère que ce sont les politiciens, par la fausseté de leurs discours, qui sont responsables de cet égarement, bien davantage que la présumée décadence de la race. Selon lui, si les peuples « ont perdu le goût de la gloire et le sentiment de leur destinée, c'est bien plus à cause des politiciens que parce qu'ils y étaient portés par la lassitude des âges, par l'indolence et par la fatigue de leur race » $(1899$, p. $89-90)$. Lemonnier prône un déconditionnement qui passe par un retour au paradis originel, mais en ajoutant au récit biblique un dépassement du péché, qui ancre les destinées humaines dans une trajectoire de vie et non pas de déclin et de mort. Il tente d'offrir une alternative à l'orthodoxie chrétienne et de conjurer le puritanisme de la Belgique de son époque, qui est la cause du profond désarroi de la jeunesse comme il l'a expliqué dans L’Homme en amour, où son personnage se lamente de ce que «notre éducation faussée, avec l'ignorance de nous-mêmes et la déviation de nos plus irrésistibles penchants, propage les pires perversions " (1901, p. 39-40). Eekhoud cherche lui aussi à émanciper ses compatriotes, à les libérer du carcan qui leur a été imposé par la Réforme protestante et la Contre-réforme en faisant appel aux ancêtres et à la nature. Il rêve de retourner au monde des origines,

15. On retrouve ici une conception du bonheur similaire à celle promue par Zola dans Fécondité par exemple, fondée sur une soumission aux élans dictés par la nature, en particulier l'instinct de procréer. Les deux écrivains proposent le même idéal d'une vie frugale passée aux côtés d'une femme représentée comme le symbole de la maternité, au milieu de nombreux enfants, près de la terre, à accomplir son devoir d'époux et de père. Toutefois, si Lemonnier fustige lui aussi la sexualité improductive, il n'est pas comme Zola hanté par la peur d'une dégénérescence de la société due à la dépopulation. Ce ne sont pas les théories de Malthus qui induisent les hommes et les femmes en erreur dans les romans naturistes de Lemonnier, mais plutôt l'engorgement des pulsions libidinales causé par les préceptes contre-nature d'un christianisme qui enseigne le mépris du corps. 
où le corps nétait l'objet d'aucun tabou. Il suit le commandement de Bouhélier, qui exhorte les poètes à " ressusciter les énergies nationales qui sommeillent dans le sein des hommes" (1899, p. 90). Les énergies qu'invoque Eekhoud sont celles des ancêtres flamands, qui appartiennent à une race paienne, fruste, désinhibée. Toutefois, si l'on retrouve chez Eekhoud, tout comme chez Lemonnier, le mythe du bon sauvage, l'auteur des Libertins d'Anvers ne fonde pas le monde auquel il rêve sur un couple formé par un homme et une femme accompagnés de leur progéniture.

La communauté idéale qu'envisage Eekhoud est une communauté dans laquelle les alliances entre les hommes et les femmes sont fluides, se faisant et se défaisant au gré de la fantaisie de chacun, et où les couples sont uniquement préoccupés par la jouissance. La procréation n'est pas mentionnée dans les descriptions que fait Eekhoud des sectes hérétiques du Moyen Âge et de la Renaissance, comme si la sexualité libre n'avait pas à s'en préoccuper. Conformément aux leçons du naturisme, la nature, dans Les Libertins d'Anvers, montre le chemin d'une libération qui réconcilie l'être humain avec le cosmos, mais cette libération passe par un tantrisme, la recherche d'une extase érotique, et n'incorpore pas la dimension familiale. Elle n'intègre pas non plus l'idée que le salut de l'homme et de la femme réside dans le travail. Dans Les Libertins d'Anvers, le travail n'occupe pas la fonction de révélateur de l'âme humaine qu'il a dans les romans de Lemonnier. Éloi appartient à la corporation des couvreurs d'ardoises, dont Eekhoud décrit le travail quotidien afin de mettre en évidence leur courage et leur agilité. Le jeune Éloi est réfractaire au métier paternel. De nature rêveuse, il préfère errer dans la ville d'Anvers à la recherche d'aventures. Il est sensible, nerveux, contemplatif, enthousiaste, tout le contraire des « tempéraments plutôt rassis et flegmatiques " auxquels convient d'habitude la profession de couvreur (1912, p. 143). Éloi est avant tout un jouisseur, un hédoniste invétéré. La vie, dans tout ce qu'elle peut offrir d'agréable aux sens, exerce sur lui une profonde attirance. Sa morale est guidée par ses goûts, ses affections, son sentiment du Beau. Éloi n’a pas le sens du devoir familial. Il sémancipe au contraire de sa famille, car celle-ci n'est pas conforme à ses penchants et entraverait son épanouissement. Il ne suit pas la voie tracée par son père, il ne devient pas lui-même couvreur et ne fonde pas de famille, mais il n'est pas pour autant immoral. Il se sent investi d'une mission d'ordre divin qui l'amène à créer la secte des Loïstes. Le personnage d'Eekhoud, " [c] e panthéiste, cet épicurien renforcé qui proclamait les droits imprescriptibles de la chair ", possède une intransigeance que lui inspire sa nature sensuelle, son sens de la justice étant dicté par son amour inconditionnel de la vie (1912, p. 252-253).

\section{Dénonciation anarchiste de l'orthodoxie religieuse et sociale}

Dans sa préface aux Libertins d'Anvers, Vaneigem considère que le combat des hérésiarques raconté par Eekhoud est celui de l’émancipation du corps et de la " réhabilitation des pulsions de vie» $(2009$, p. 12). Vaneigem rapproche ce combat contre 
le totalitarisme religieux de celui de l'homme d'aujourd'hui contre un capitalisme déshumanisant qui force l'être humain à la survie plutôt qu'à la vie et l'asservit au nom du rendement et de l'efficacité motivés par l'appât du gain. L'intérêt que des philosophes anarchistes, tel que Vaneigem ou encore Onfray, portent aux hérésiarques du Moyen Âge et de la Renaissance tient au fait que ceux-ci représentent un point d’ancrage dans l'histoire, à partir duquel il est possible d'imaginer une société à venir dans laquelle l'être humain serait libéré du carcan de la religion, des hiérarchies de classes sociales et de sexes, de la propriété privée, du couple et de la famille ${ }^{16}$. C'est la perspective d'une semblable libération qui amène Eekhoud à écrire sur des épisodes de l'histoire de sa ville natale, cette évocation lui permettant de se projeter dans un passé fantasmé et de rêver une libération radicale des mœurs qui rendrait possible l'acceptation par le plus grand nombre de son orientation sexuelle. Car sa vaste fresque historique qui retrace les hérésies anversoises met en valeur des comportements contraires à l'ordre social dans l'espoir d'encourager une manière de voir plus ouverte, plus tolérante, et surtout moins hostile à l'homosexualité. Eekhoud développe dans Les Libertins d'Anvers un discours anarchisant, dont nous proposons de préciser les enjeux en nous appuyant sur les travaux de Vaneigem, Onfray et Fourier.

Eekhoud passe en revue dans son roman les principaux mouvements hérétiques qui s'opposèrent à la doxa de l'Église romaine, à savoir les Vaudois, les Kloeffers, les Turlupins, les Adamites, les Hommes de l'Intelligence, les sectes fondées par Tanchelin d'Anvers et Guillaume Cornélis, et enfin les Loïstes. Il insiste sur la libération des mours que revendiquent ces mouvements, une libération fondée sur l'abandon du péché et la célébration des instincts voulus par la nature et par Dieu. La nudité et la pratique de la polygamie sont célébrées par ces groupes de réfractaires qui ne croient pas à l'enfer. Pour eux, le but de la vie est la poursuite d'un bonheur charnel. Comme le souligne Vaneigem, ces libertins spirituels « vivaient dans la quête de l'amour et y découvraient le sens même de leur existence» (1993, p. 365). Éloi, par exemple, propage « sa conception d'une vie inspirée par un Dieu bon, hostile à la violence, aux châtiments, à la culpabilité et dont la grâce rend l'innocence édénique à qui

16. Onfray décline son credo anarchiste dans un court essai posté sur son site internet la semaine du 3 avril 2021. Il y fustige Marine Le Pen pour son ignorance et son manque de convictions. Il l'oppose à son père, l'ancien leader du Front national, qui avait selon lui le mérite de tenir un discours authentique. Cette opposition, qui semble mettre en valeur Jean-Marie Le Pen et pourrait être interprétée comme un glissement d'Onfray vers l'extrême droite, n'est qu'un outil rhétorique. Onfray n'est favorable ni au père ni à la fille. Ce qu'il critique à travers Marine Le Pen, c'est une politique sans fondements, qui ne fait que duper les électeurs. Onfray affirme surtout sa volonté de rester en-dehors des " cohortes " et de ne prendre parti que pour les opprimés : « Je ne suis pas de ceux qui hurlent avec les loups. [...] Je ne mêle pas ma voix au chœur des pourvoyeurs d'échafauds. [...] Je ne participe jamais au quart d'heure de haine décidé par Big Brother " (2021). En filigrane du portrait qu'il brosse de Jean-Marie Le Pen se dessine une ligne de pensée guidée par un souci de cohérence avec soi-même. Nous ne sommes pas loin de l'intransigeance du héros eekhoudien, dont Henry de Kehlmark est le porte-parole lorsqu'il déclare : «Oui, rester moi-même [...] ! Ne pas changer ! [...] Demeurer fidèle jusqu'au bout à ma nature juste, légitime!» (1899, p. 260-261). 
suit ses désirs et sa propension au bonheur " (Vaneigem, 1993, p. 366). Onfray voit en lui l'instigateur d'une révolution radicale, qui fixe l'hédonisme comme « impératif catégorique » :

D’une manière presqu'ironique, Éloi formule l'impératif catégorique hédoniste en parodiant saint Paul. On connait l'enseignement de l'hystérique de Tarse : ne pas faire à autrui ce que l'on ne voudrait pas qu'il nous fasse. Cette morale négative prescrit un évitement. Le philosophe couvreur va au-delà et affirme, dans une phrase considérable, qu'il s'agit désormais, dans le Libre-Esprit et au-delà, de considérer comme l'impératif catégorique hédoniste : faire à autrui ce que l'on voudrait qu'il nous fasse. (2006, p. 133)

Onfray fait ici référence à la phrase d'Éloi dans sa lettre aux réformés d'Anvers : « la nature m'enseigne à faire à mon prochain ce que je voudrais qu'il me fit » (Vaneigem, 1993, p. 366). Selon Éloi, les hommes qui font preuve d'une telle sympathie envers leurs semblables agissent sous l'impulsion du Saint-Esprit et sont guidés par leur foi en Dieu.

Les travaux de Vaneigem et d'Onfray nous renseignent sur le fait que l'épicurisme des partisans du Libre-Esprit va de pair avec la sanctification de la pauvreté et un certain féminisme. Dès le début du XII ${ }^{e}$ siècle apparait avec Tanchelin " un renouveau de la communauté chrétienne, hostile aux richesses et au faste inutile de l'Église, et identifiant la véritable pratique apostolique à la pauvreté, à la fraternité, à la solidarité organisée par les œuvres d'entraide et de secours aux affamés " (Vaneigem, 1993, p. 258). L'idée du partage des richesses et de la sanctification de la pauvreté sera reprise par la suite par un grand nombre d'hérésiarques. Les Loïstes, par exemple, pratiquent un égalitarisme qui les amène à partager leurs biens, les plus riches se trouvant ainsi libérés du péché. Vaneigem explique que «[p]armi les loïstes, les pauvres accèdent $[\ldots]$ à une existence sans souci pécuniaire, à la faveur d'une prise de conscience où les riches se livrent aux plaisirs sans avoir désormais à redouter ni les remords, ni le péché, ni les angoisses des possédants, ni le ressentiment des dépossédés » (1986, p. 207). Pour les hérésiarques, la pauvreté est une vertu évangélique qui place les hommes dans le sein de Dieu. Eekhoud souligne le partage des richesses qui met les membres de la secte des Loïstes sur un pied d'égalité, cet égalitarisme remettant en cause la structure d'une société fondée sur des inégalités :

Loïet [Éloi] avait violé les conventions par lesquelles les mortels se créent des supériorités impies et factices : les privilèges de la richesse et de la naissance, ou du moins les avaitil dépouillés de leur prestige en les ravalant, en les subordonnant aux dons naturels, aux valeurs réelles, en les mettant à la portée des élites, des génies et même des simples bonnes âmes, en supprimant toute démarcation sociale. (1912, p. 376)

Le communautarisme des mouvements hérétiques encourage non seulement le partage des richesses, mais aussi le respect du corps de la femme et son droit à une 
sexualité libre. Tanchelin "favorise les mariages "selon le cœur" " (Vaneigem, 1993, p. 257). Vaneigem explique que les Vaudois « reconnaissent à la femme les mêmes droits que l'homme " (1993, p. 278). Guillaume Cornélis, quant à lui, limite pour les couples le nombre des rapports sexuels à trois fois par semaine afin d'établir un code de courtoisie où la femme n'est plus l'objet des constantes sollicitations de l'homme. Dans la secte des Loïstes, comme le précise Eekhoud dans Les Libertins d'Anvers, les femmes ont le droit de se donner ou de se refuser aux hommes qui recherchent leurs faveurs, ayant ainsi le contrôle de leur corps.

Au-delà des dogmes de l'Église, c'est la propriété privée, source de l'exploitation de l'homme par l'homme et du pouvoir arbitraire d'un sexe sur l'autre, qui est questionnée par les mouvements hérétiques, au nom de Dieu, de la nature et du plaisir. La jouissance réveille un instinct qui décille les yeux des hommes en leur faisant entrevoir le bonheur auquel ils ont droit. Les murs que tentent d'ériger les dominants afin de garder la main mise sur une partie de la population, les classes laborieuses, les femmes, s'effondrent pour faire place à un horizon qui n'est plus borné ni par le péché, ni par l'enfer, ni par les interdits imposés à tous ceux qui font les frais d'une exploitation systématique. En faisant le récit des prises de position radicales d'hérésiarques qui sapent les bases d'une société gouvernée par l'Église et par l'argent, Eekhoud vient nourrir le discours des anarchistes du début du $\mathrm{XX}^{e}$ siècle ${ }^{17}$. Dans sa préface aux Libertins d'Anvers, Vaneigem identifie clairement l'anti-bourgeoisisme sans concessions dont fait preuve Eekhoud lorsqu'il décline page après page la manière dont ses compatriotes ont tenu tête à l'oligarchie au fil des siècles avec une persistance héroïque. Toutefois, comme le souligne Caroline Granier, la critique que formule Eekhoud de l'aliénation des hommes par une société capitaliste qui soumet les corps jusque dans les aspects les plus intimes de leur vie a pour but un élargissement bien particulier du réel. Évoquant Escal-Vigor, Granier explique : « une vision utopique est à l'œuvre dans le roman, vision qui contribue à lutter contre les règles d'une société fondée exclusivement sur le profit, et imposant une sexualité normative et aliénante » (2003). Elle précise qu'Eekhoud, en écrivant un roman qui met en scène une relation amoureuse entre deux hommes, "déplace les frontières entre l'utopie et le réel » (2003). Ces remarques sont applicables à l'ensemble de l'œuvre d'Eekhoud, qui cherche à transmettre une vision plus large, une autre manière de voir qui bouleverse l'orthodoxie sociale et permette de porter un regard apaisé sur une forme de sexualité, l'homosexualité, considérée comme une déviance.

L'homoérotisme est omniprésent dans Les Libertins d'Anvers. Il apparaît dansla description des jeunes hommes " décoratifs " qui composent l'entourage de Tanchelin, des Vaudois « sensuels et de chair débridée » qui pratiquent « la plus large fraternité », des Hommes de l'Intelligence qui considèrent que « Dieu exige de nous toutes

17. Sur la situation d'Eekhoud par rapport aux mouvements anarchistes de son époque, se reporter aux pages du livre de Granier (2008) consacrées à l'auteur des Libertins d'Anvers. 
les passions charnelles, même les plus décriées ", des cérémonies d'initiation inventées par Éloi au cours desquelles « il appariait le gentilhomme et le gueux, substituant les haillons de l'un aux précieuses nippes de l'autre » et où « [d]es unions fraternelles se consommaient » $(1912$, p. $319,65,75,322)$. Il y a aussi la relation ambiguë entre Éloi et son ami et futur bourreau Peer de Breeder, relation qui est au cœur du roman d'Eekhoud et est chargée d'une forte sensualité. Sous couvert d'égalitarisme et de fraternité, Eekhoud évoque des fantasmes familiers portant sur des hommes du peuple, prolétaires et sous-prolétaires, qui sont les sujets de prédilection de sa rêverie érotique, celle-ci influant sur la manière dont l'écrivain représente les hérésies qui se développèrent dans sa ville natale ${ }^{18}$.

Afin de défendre de façon détournée les amours homogéniques, Eekhoud se fait le chantre de l'amour libre à travers la glorification de la sexualité égalitaire et désinhibée pratiquée par les partisans des mouvements hérétiques qui prolifèrent à Anvers dès le haut Moyen Âge. En prenant parti pour la jouissance illimitée du corps, il se situe dans la lignée de Fourier, qui fustige « la constance et la fidélité, si incompatibles avec le vœu de la nature et si fatigantes aux deux sexes, que nul être ne s'y soumet quand il jouit d'une pleine liberté » (Guérin, 1975, p. 59). Fourier qualifie l'exclusivisme du couple d'" égoïsme amoureux ", affirmant que "les hommes aiment tous la polygamie et [...] les dames civilisées tant soi peu libres aiment de même la pluralité d'hommes » (Guérin, 1975, p. 90, 187). Pour lui, le mariage et la famille ont pour but de canaliser l'énergie du célibataire et de le contraindre à devenir un membre productif de la société. Fourier dénonce le caractère contre-nature de la monogamie, qui mène inévitablement à l'adultère, et la victimisation des femmes à laquelle tend la répression sexuelle, la jeune fille étant traitée dans le mariage comme un bien que l'on s'approprie. Eekhoud rejoint Fourier dans l'idée d'encourager l'épanouissement de l'individu par la jouissance et le plaisir des sens. Toutefois, alors que pour Fourier le fait de favoriser l'expression des passions sert à maximiser l'engouement au travail, l'hédonisme que prône Eekhoud n'est pas rattaché à des fins utilitaires. Comme Fourier, Eekhoud souligne le caractère malfaisant de l'engorgement des passions, mais tandis que pour Fourier le développement harmonieux de celles-ci passe par une organisation sociale visant à accroître l'efficacité de la participation de chaque individu au bien-être collectif, pour Eekhoud il s'agit d'un développement spontané, la seule injonction étant de suivre son plaisir tout en respectant son prochain. En cela, la pensée d'Eekhoud est voisine de celle des partisans de la révolution sexuelle qui redécouvrent Fourier à partir des années 1960 et offrent une lecture sélective de son œuvre, voyant dans l'auteur du Nouveau monde amoureux un précurseur de la libération sexuelle ${ }^{19}$.

18. Sur l'importance des fantasmes homosexuels dans l'œuvre d'Eekhoud, voir l'article d'Aron (1982) qui met en évidence le fait que ces fantasmes conditionnent l'engagement politique de l'écrivain et se rattachent à la structure profonde de son œuvre. Voir aussi mon article sur paysans, ouvriers et voyous dans l'œuvre d'Eekhoud (Chavasse, 2004).

19. Michel Bozon remarque que Fourier est utilisé à l'époque de la révolution soixante-huitarde 
Eekhoud se rapproche de Fourier dans sa critique d'une forme d'amour dit civilisé qui repose sur la contrainte du mariage, mais la philanthropie amoureuse que prêche ce dernier fait place dans Les Libertins d'Anvers à un panthéisme, une communion entre les êtres conforme à la nature, qui n'obéit à aucune rationalité et prend volontiers la forme d'une débauche carnavalesque.

Léclatement du couple et de la famille, piliers de la société bourgeoise, et la promotion de la polygamie sont au cœur des Libertins d'Anvers, contribuant à faire de ce roman une œuvre profondément subversive, foncièrement distincte des romans naturistes de Lemonnier, qui prêchent au contraire une monogamie indispensable au confort physique et moral de l'homme et à la régénération de la race. L'hédonisme et l'amour libre sont clairement réprouvés par Lemonnier, qui les associe à la décadence de la société. À la relation monogame des couples d'Adam et Ève et $A u$ coeur frais de la forêt, l'écrivain oppose la jouissance infructueuse du protagoniste de L'Homme en amour, qui se perd dans une sexualité débridée, sans amour et sans espoir, qui le laisse exsangue, rongé par un désir insatiable. Dans les romans naturistes de Lemonnier, même si l'union de l'homme et de la femme est respectueuse du plaisir de chacun et laisse à la femme son libre arbitre, le rapprochement des corps doit être productif en vertu de l'injonction de la nature à procréer, et cet impératif de productivité impose à l'homme le choix d'un type particulier de femme qui soit conforme à ses besoins. Cette représentation de la femme aboutit à une aliénation, puisque celle-ci ne peut exister qu'en tant que compagne et que mère. Chez Eekhoud, le rôle de la femme est également de faciliter le rêve de bonheur poursuivi par un homme. La femme est certes glorifiée dans Les Libertins d'Anvers, comme dans les romans de Lemonnier, mais la fonction qu'elle occupe fait d'elle un instrument au service d'une vision masculine de la communauté idéale.

\section{La place de la femme : vierge salvatrice ou sacrifiée}

Dans Adam et Ève, la femme est adulée pour son instinct infaillible, sa compréhension innée de la nature et de la destinée humaine. Adam déclare à propos de la femme qui partage sa vie, Ève : " elle était bien plus avancée que moi aux secrets de l'univers. J’étais devant elle comme les bergers sous l'étoile» $(1899$, p. 185). Ėve est pleine de bon sens. Elle pousse Adam à se focaliser sur l'essentiel. C'est elle qui le ramène dans le droit chemin, lui dicte son comportement et l'empêche de sombrer dans la violence et la fornication. Le personnage de Frilotte dans Au coeur frais de la forêt est plus ambigu. Ève est une femme plantureuse, faite pour enfanter. Frilotte,

comme " un Nostradamus de la libération sexuelle " (2005, p. 11). Bozon précise qu'il existe dans la lecture libertaire de Fourier que font, à la suite de Daniel Guérin, des philosophes comme Vaneigem ou Onfray, une contradiction « entre l'appel à la liberté amoureuse et le caractère extrêmement organisé de la société que Fourier propose» (2005, p. 12). 
au contraire, est maigre et ressemble à un garçon. L'une vient de la saine campagne, l'autre a grandi dans une banlieue interlope. Ève est innocente et droite, alors que Frilotte est malicieuse et imprévisible. Pourtant, tout comme Ève, Frilotte guide son compagnon et l'empêche de s'égarer. C'est elle qui ramène Petit Vieux dans la forêt et l'incite à tourner définitivement le dos à la société. Frilotte sait intuitivement que la vraie vie doit se dérouler au sein de la nature. Ėve et Frilotte sont comme des pythies qui permettent à l'homme de se retrouver et de se régénérer. Ce sont elles qui assurent la pérennité de son rêve. Leur âme est simple, imperméable aux tourments. Lorsqu'Adam et Petit Vieux se trouvent tous deux rongés par la jalousie, leurs jeunes compagnes viennent à leur secours. Elles les réconfortent, car elles possèdent les clés de l'amour, cette certitude du cœur sur laquelle reposent les unions heureuses.

Toutefois, bien qu'elle soit à l'origine de la constance du couple et que son tempérament s'accorde avec un retour à la nature, la femme n'est que la facilitatrice de l'utopie dans les romans naturistes de Lemonnier. Elle n'en est pas le principal architecte. L'homme a surtout besoin d'elle pour ne pas se perdre et pour accomplir son devoir naturel, c'est-à-dire procréer. Lemonnier ne raconte pas l'accomplissement de destins féminins. Ses personnages de femmes sont des instruments mis au service de fantasmes masculins. L'idylle bucolique n'est possible chez Lemonnier qu'avec un type particulier de femme, la vierge qui n'a pas été corrompue par une sexualité improductive. Cette femme-là ne pose aucun danger pour l'homme. Elle est comme une source d'eau pure à laquelle celui-ci peut se désaltérer sans prendre de risque. Au contraire, la femme dont l'instinct sexuel a été inutilement éveillé devient une sorte d'hystérique qui entraîne ses amants dans une sexualité infernale. Aude dans L'Homme en amour est l'archétype de cette femme diabolique qui conduit l'homme à sa perte. Comme le note Sylvie Thorel-Cailleteau, elle est « la mère porteuse de mort, [...] une sorte de vampire qui 'porte à sa ceinture le signe de la Bête' » (1994, p. 443). La présence de cette femme possédée rend impossible le retour au Paradis originel auquel aspire le protagoniste de L'Homme en amour. Celui-ci a peur d'être contaminé par l'instinct sexuel de la femme, comme si son hystérie pouvait lui être transmise.

Afin de conjurer le mal, Adam choisit parmi les jeunes filles qu'il rencontre à l'orée de la forêt la seule qui lui semble n'avoir jamais eu de rapports sexuels, celle " dont la voix avait un charme d'enfance» $(1899$, p. 8). Le couple formé par Frilotte et Petit Vieux est, dans un premier temps, un couple fraternel. Aux yeux de son compagnon, Frilotte est un être asexué. Petit Vieux la protège et apprend à l'aimer comme un frère avant d'éprouver pour elle du désir. Leur union évolue naturellement de l'innocence de l'enfance à des ébats amoureux qui aboutissent très vite à l'enfantement. Les bases du couple ne sont pas souillées par une sexualité effrénée. Le plaisir reste maîtrisé, le désir ne s'emballe pas. Dans Adam et Ėve, l'homme et la femme connaissent une période d'euphorie sexuelle, mais cet abandon est décrit comme l'initiation à une sensualité qui les régénère en les rapprochant de la nature. Elle leur permet de prendre conscience de leurs corps et de communier avec les éléments qui les entourent. Dans Lîle vierge, les femmes sont jeunes comme Frilotte et innocentes 
comme Ève. Elles entretiennent avec les hommes dont elles s'éprennent des liens fraternels. Sylvan et Élée sont cousins et ont été élevés en frère et sœur. Éleuthère et Hylette sont également cousins. Leurs amours restent platoniques. Enfant d'une relation incestueuse, Élée est marquée par une hérédité qui fait d’elle une femme dangereuse, mais elle disparait à la fin du roman avant d'avoir pu pervertir Sylvan. L'autre femme menaçante dans L'Île vierge est Cordalie, la mère d'Élée, qui vit dans un couvent depuis la naissance de sa fille et se complaît dans une volupté extatique proche de la folie. Cordalie est le prototype de la femme hystérique, dévorée par son instinct sexuel, qui doit être mise à l'écart de la société. Elle est l'antithèse des femmes novices qui offrent à leurs partenaires la pureté nécessaire pour garantir la réalisation de leur rêve de bonheur.

Alors que Lemonnier met en avant des personnages de vierges salvatrices, chargées de préserver les protagonistes masculins contre l'hystérie contagieuse des femmes et de permettre aux mondes rêvés de se construire sur le socle de la famille et du couple monogame, Eekhoud sacrifie ses personnages de femmes dans le déploiement d'une utopie communautaire dont elles sont les victimes. Ce qui intéresse prioritairement Eekhoud dans les hérésies du Moyen Âge et de la Renaissance, c'est leur permissivité à l'égard d'une sexualité libre qui lui sert de subterfuge pour évoquer des rapprochements de corps d'hommes. La sexualité mise en valeur dans Les Libertins d'Anvers est centrée sur les personnages masculins. Certes, ceux-ci ne sont pas tous homosexuels, mais Eekhoud entretient le trouble quant à leurs pratiques sexuelles. Éloi, par exemple, est attiré par Peer, qu'il trouve " atroce, mais très beau tout de même ", tout en ayant des rapports sexuels avec un grand nombre de femmes (1912, p. 179). Surtout, le regard porté sur les corps masculins est empreint d'homoérotisme. Dans les rares descriptions des corps féminins, ceux-ci sont célébrés pour leur perfection plastique, leur beauté froide et saine, comme les corps des jeunes bourgeoises et de Dillette qui "déferl[e]nt en vagues lactées " lors de la procession organisée en l'honneur de la première visite de Charles Quint à Anvers (1912, p. 236).

La femme qui se sacrifie pour défendre les convictions de l'homme qu'elle aime est une figure récurrente dans l'œuvre d'Eekhoud. Dans Les Libertins d'Anvers, le personnage de Dillette, "s'ingéniant à détourner l'orage de la tête de son amant ", accepte de se donner à Peer afin de sauver la secte des Loïstes (1912, p. 342). Dillette, dans les yeux de qui Loïet lit « tant de splendeur morale, de religion pour lui, d'ineffable amour ", rappelle le personnage de Blandine dans Escal-Vigor, qui est prête à mourir pour Henry, son compagnon homosexuel, en professant son soutien aux amours homogéniques (1912, p. 343). Un autre personnage de femme sacrifiée dans Les Libertins d'Anvers est Mariette Eymhof, qui a été abandonnée par son mari, Germain Bousseraille, converti au Loïsme. Cette épouse éconduite a choisi non pas de soutenir la foi de son mari, mais de la conspuer. Éloi néprouve que du mépris pour cette femme prosaïque. Contrairement aux personnages de femmes éthérées que sont Dillette et Blandine, Mariette représente la femme utilitaire qui fait tomber l'homme dans les filets de la famille. En la voyant flanquée de ses deux fils, Éloi, dans un geste 
caractéristique de son refus de toute entrave sociale et familiale, et conformément à la tonalité homoérotique du roman d'Eekhoud, " n'opposa qu'une moue dédaigneuse à la mégère, mais il caressa d'un ineffable sourire les deux jolis petiots en lesquels il revoyait l'image fervente et féale du bon rustre, leur père » (1912, p. 381).

Tout comme Eekhoud, Lemonnier rêve de régénérer ses compatriotes, de les libérer du carcan imposé par une civilisation mensongère. Le regard des deux écrivains est tourné vers le passé, un âge d’or de l'humanité, le jardin d'Éden ou une période de l'histoire caractérisée par une renaissance païenne, où « l'Homme régénéré aurait reconquis la bonté première " (Rency, 1922, p. 43). L'acquisition de cette bonté primordiale repose sur un dépassement du péché. Lemonnier met en scène dans Adam et Ève et Au cour frais de la forêt une utopie qui finit bien parce que les personnages ont réussi à contrôler leurs instincts et ont pu s'ouvrir à une bonté évangélique figurée par une communion avec la création tout entière. L'attitude d'Eekhoud à légard de la part d'ombre de l'être humain est plus ambiguë que celle de Lemonnier. Dans Les Libertins d'Anvers, le péché est du côté de Peer, dont l'agressivité trouble Éloi. Il existe entre les deux hommes une tension sexuelle faite d'une attirance sadomasochiste. Eekhoud ne liquide pas l'instinct sanguinaire de la race, contrairement à Lemonnier. Il en fait une composante du désir homosexuel. L'appel à un renouveau de la société que sous-tendent les romans de Lemonnier et d'Eekhoud s'appuie sur deux visions qui, malgré un fond commun rattaché à la quête identitaire des artistes belges de l'époque, ne sont pas novatrices de manière égale en matière de sexualité. Le droit à une sexualité libre revendiqué par Eekhoud s'oppose à l'aspiration traditio-

naliste de Lemonnier à une monogamie salvatrice. Les deux écrivains se retrouvent néanmoins dans la manière qu'ils ont de composer une fantasmagorie masculine qui, en dépit d'un élan égalitaire, tend à instrumentaliser la femme.

\section{RÉFÉRENCES}

Aron, P. (1985). Les Écrivains belges et le socialisme (1880-1913). L'expérience de l'art social: d'Edmond Picard à Émile Verhaeren. Bruxelles : Labor.

Aron, P. (1982). Structures et significations idéologiques de l'œuvre romanesque de Georges Eekhoud (1854-1927). Réseaux, 39-40, 3-18.

Bazalgette, L. (1904). Camille Lemonnier. Paris : Bibliothèque internationale dédition,

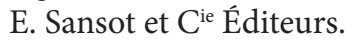

Bouhélier, S.-G. de. (1899). Les Éléments d’une renaissance française. Paris : Bibliothèque artistique et littéraire.

Bouhélier, S.-G. de. (1900). Au cour frais de la forêt (critique). La Revue naturiste, 4, 252-254. Bozon, M. (2005). Fourier, Le Nouveau monde amoureux et mai 1968. Politique des passions, égalité des sexes et science sociale. Clio, 22, 123-149. http://journals.openedition.org/clio/1758

Chavasse, P. (2004). Paysans, ouvriers et voyous dans l'œuvre de Georges Eekhoud. Excavatio : Emile Zola and Naturalism, 19, 213-222. 
Chavasse, P. (2022). Terre, amour et morts dans L'Autre vue de Georges Eekhoud. French Forum, 46(3). (à paraître).

Christie, J. (1963). Naturalisme et naturisme. Les relations d'Émile Zola avec Saint-Georges de Bouhélier et Maurice Leblond. Nottingham French Studies, 2, 11-24.

Citti, P. (1987). Contre la décadence. Histoire de l'imagination française dans le roman 18901914. Paris : PUF.

Detemmerman, J. (1984). Le Procès d'Escal-Vigor. Dans R. Trousson (dir.), Le Naturalisme et les lettres françaises de Belgique (p. 141-169). Bruxelles: Université de Bruxelles.

Des Ombiaux, M. (1909). Camille Lemonnier. Bruxelles : Charles Carrington.

Dessy, C. (2016). Le naturalisme au service du roman historique : Les Libertins d'Anvers de Georges Eekhoud (1912). Les Cahiers naturalistes, 90, 217-232.

Dessy, C. (2020). Georges Eekhoud, éthologue du siècle de Shakespeare. Textyles, 58-59, 169196.

Dumont, G.-H. (1991). La Belgique. Paris : Presses Universitaires de France.

Dupont, W. (2015). Free-Floating Evils: A Genealogy of Homosexuality in Belgium. Antwerp : University of Antwerp.

Eekhoud, G. (1899). Escal-Vigor. Paris : Mercure de France.

Eekhoud, G. (1912). Les Libertins d’Anvers. Légende et histoire des Loïstes. Paris : Mercure de France.

Gonne, M. (2017). Contrebande littéraire et culturelle à la Belle Époque : Le "hard labour» de Georges Eekhoud entre Anvers, Paris et Bruxelles. Louvain : PUL.

Gorceix, P. (1997). La Belgique fin de siècle. Bruxelles : Complexe.

Gorceix, P. (1998). Fin de siècle et symbolisme en Belgique. Bruxelles : Complexe.

Granier, C. (2008). Les Briseurs de formules. Les écrivains anarchistes en France à la fin du XIX siècle. Cœuvres-et-Valsery : Ressouvenances.

Granier, C. (2003). Utopie sexuelle : Escal-Vigor. R.A. Forum. Thèses et mémoires. http://archive.wikiwix.com/cache/index2.php?url=http\%3A\%2F\%2Fraforum.info\%2Fdissertations\%2Fspip.php\%3Farticle76

Guérin, D. (1975). Vers la liberté en amour, de Charles Fourier. Paris : Gallimard.

Krzywkowski, I. (1997). Camille Lemonnier : du "Jardin de la mort » au rêve de l'âge d'or. Naturalismes, Nord, revue de critique et de création littéraires du nord, 30, 17-28.

Lemonnier, C. (1896). L'̂̂le vierge. Paris : Dentu.

Lemonnier, C. (1899). Adam et Ėve. Paris : Ollendorff.

Lemonnier, C. (1900). Au cour frais de la forêt. Paris : Ollendorff.

Lemonnier, C. (1901). L'Homme en amour. Paris : Ollendorff.

Lemonnier, C. (1907). Préface. Dans S.-G. de Bouhélier, Choix de pages anciennes et nouvelles (p. 9-20). Bruges : Arthur Herbert.

Luc, A.-F. (1990). Le Naturalisme belge. Bruxelles : Labor.

Lucien, M. (1999). Eekhoud le rauque. Lille : Presses Universitaires du Septentrion.

Martin-Schmets, V. (1984). Le naturisme en Belgique de Vandeputte au Congrès de La Lutte. Dans R. Trousson, Les Relations littéraires franco-belges de 1890 à 1914 (p. 65-76). Bruxelles : Éditions de l'Université de Bruxelles.

Onfray, M. (2006). Contre-histoire de la philosophie II. Le Christianisme hédoniste. Paris : Grasset. Onfray, M. (2021). Le Front national est mort. https://michelonfray.com/interventions-hebdomadaires/le-front-national-est-mort? mode=video 
Quaghebeur, M. et Labio, C. (2002). The Sixteenth Century: A Decisive Myth. Yale French Studies, 102, 115-141.

Quaghebeur, M. (2005). Le XVI siècle : un mythe fondateur de la Belgique. Textyles, 28, 30-45. Rency, G. (1922). Camille Lemonnier. Bruxelles : La vie intellectuelle.

Rosenfeld, M. (2018). Gay Taboos in 1900 Brussels : The Literary, Journalistic and Private Debate Surrounding Georges Eekhoud's Novel Escal-Vigor. Dix-neuf, 22, 98-114.

Soncini, A. (2004). La médiévalisation de la littérature belge de langue française, un archétype flamand. Cahiers de Recherches Médiévales et Humanistes, 11, 151-164.

Thorel-Cailleteau, S. (1994). La Tentation du livre sur rien : naturalisme et décadence. Montde-Marsan : Éditions interuniversitaires.

Vaneigem, R. (1986). Le Mouvement du Libre Esprit. Paris : Ramsay.

Vaneigem, R. (1993). La Résistance au christianisme. Les hérésies des origines au XVIII siècle. Paris : Fayard.

Vaneigem, R. (2009). Préface. La vraie vie nous dispense des religions. Dans G. Eekhoud, Les Libertins d'Anvers (p. 7-16). Bruxelles : Aden.

RÉSUMÉ : Dans les dernières années du XIX siècle, l'écrivain belge Camille Lemonnier publie trois romans, L'̂lle vierge, Adam et Ève, et Au cour frais de la forêt, qui véhiculent le rêve de voir l'humanité libérée du carcan imposé par une société qui asservit l'homme et la femme et dénature leur instinct. Le Belge Georges Eekhoud publie en 1912 Les Libertins d'Anvers, qui retrace l'histoire des hérésies chrétiennes à Anvers du XII ${ }^{\mathrm{e}}$ siècle jusqu’à leur répression par la Réforme protestante et la Contre-réforme. Nourris par les mêmes préoccupations identitaires, Lemonnier et Eekhoud proposent des modèles de communautés utopiques qui s'inspirent à la fois du paganisme et de l'évangélisme chrétien. Les deux écrivains font l'apologie de la charité et du respect du prochain et de la nature. Toutefois, ils diffèrent dans l'intérêt qu'ils accordent au couple et à la famille comme fondement social, Lemonnier appliquant les leçons du naturisme, tandis qu'Eekhoud se situe davantage dans un courant de la pensée anarchiste représenté notamment par Charles Fourier, Raoul Vaneigem et Michel Onfray.

Mots-clés : naturisme, fin de siècle, Belgique, paganisme, hérétiques, Camille Lemonnier, Georges Eekhoud

\section{Two visions of happiness according to nature in the works of Camille Lemonnier and Georges Eekhoud}

ABSTRACT: In the last years of the 19th century, the Belgian writer Camille Lemonnier published three novels, L'IleVierge, Adam et Ève, and Au cour frais de la forêt, which conveyed the dream of seeing humanity freed from the shackles imposed by society that enslaves men and women and distorts their instincts. The Belgian Georges Eekhoud published in 1912 Les Libertins d'Anvers, which traces the history 
of Christian heresies in Antwerp from the 12th century until their repression by the Protestant Reformation and the Counter-Reformation. Inspired by the same identity concerns, Lemonnier and Eekhoud offer models of utopian communities that draw inspiration from both paganism and Christian evangelism. The two writers praise charity, and respect for others and for nature. However, they differ in the interest they place in the couple and the family as a social foundation, Lemonnier applying the lessons of naturism, while Eekhoud is more in line with anarchist thinkers such as Charles Fourier, Raoul Vaneigem, and Michel Onfray.

Keywords: naturism, fin de siècle, Belgium, paganism, heretics, Camille Lemonnier, Georges Eekhoud 